\title{
Eficácia do resfriamento da pele no alívio da dor desencadeada pela injeção de toxina botulínica tipo A nas distonias faciais
}

\author{
Skin cooling efficacy on pain relief in periocular injections \\ with botulinum toxin $A$ in facial dystonias
}

Paula Barros Bandeira de Mello Monteiro', Nilson Lopes da Fonseca Júnior², José Ricardo Carvalho Lima Rehder ${ }^{3}$

\begin{abstract}
Resumo
Objetivo: Avaliar a eficácia do resfriamento da pele com gelo no alívio da dor desencadeada pela injeção de toxina botulínica tipo A na região periocular em pacientes portadores de distonia facial. Métodos: Neste estudo prospectivo, 13 pacientes receberam injeção de toxina botulínica tipo A em região glabelar (m. prócero) e periocular (m. orbicular) para tratamento de distonia facial. Antes das aplicações, um lado da região glabelar foi resfriado com gelo durante 5 minutos, enquanto no outro lado foi aplicada pomada Epitezan ${ }^{\circledR}$, funcionando como placebo. A aplicação foi feita primeiramente no lado resfriado. Após a aplicação em cada um dos lados os pacientes foram instruídos a dar uma nota para a dor desencadeada pela injeção, em uma escala de 0 a 10 onde 0 era ausência de dor e 10 a dor mais intensa. Resultados: A média das notas dadas pelos pacientes à dor desencadeada pela injeção no lado onde foi aplicado placebo foi 3,92 $\pm 3,28$. No local onde foi aplicado gelo a média das notas foi de 2,92 $\pm 2,18(p<0,0166)$. A média da diferença entre notas dos dois grupos foi de 1,0. Conclusão: No presente estudo o resfriamento da pele com gelo foi eficaz no alívio da dor desencadeada pela aplicação de toxina botulínica tipo A na região periocular em pacientes portadores de distonia facial.
\end{abstract}

Descritores: Distonia facial/ terapia; Toxina botulínica tipo A/uso terapêutico; Dor

\begin{abstract}
Purpose: To evaluate the efficacy of skin cooling with ice on pain relief in periocular injection with botulinum toxin type A in patients with facial dystonias. Methods: In this prospective study, 13 patients received botulinum toxin type A injection in glabela (procerus m.) and periocular region (orbicular m.) for facial dystonias treatment. Before the injections, one side of the glabela was submitted to a 5-minute cooling period, while the opposite side had Epitezan ${ }^{\circledR}$ cream applied, as a placebo. The application was done at the cooled side first. After the application on each side the patients were instructed to rate the pain associated with the injection on a scale from 0 to 10, with 0 indicating no pain and 10 the worst pain. Results: The average pain score on the side where cold was applied was 3,92 $\pm 3,28$, while on the control side the average pain score was 2,92 $\pm 2,18$ ( $p<0,0166)$. Conclusion: In this study, skin cooling with ice cubes was efficient in pain relief in periocular botulinum toxin injections in patients with facial dystonias.
\end{abstract}

Keywords: Facial dystonia/therapy; Botulinum toxin/therapeutic use; Pain

\footnotetext{
${ }^{1}$ Setor de Plástica Ocular e Vias Lacrimais da Disciplina de Oftalmologia da Faculdade de Medicina do ABC (FMABC) - Santo André (SP) Brasil;

${ }^{2}$ Setor de Plástica Ocular e Setor de Órbita da Disciplina de Oftalmologia da Faculdade de Medicina do ABC (FMABC) - Santo André (SP) - Brasil;

${ }^{3}$ Disciplina de Oftalmologia da Faculdade de Medicina do ABC (FMABC) - Santo André (SP) - Brasil.

Trabalho desenvolvido na Disciplina de Oftalmologia da Faculdade de Medicina do ABC (FMABC) - Santo André (SP) - Brasil.
}

Os autores declaram não haver conflitos de interesse

Recebido para publicação em: 29/8/2011 - Aceito para publicação em: 25/4/2012

Rev Bras Oftalmol. 2012; 71 (6): 364-7 


\section{INTRODUÇãO}

A s distonias musculares da face são caracterizadas por fechamento espasmódico e involuntário das pálpebras e evoluem com aumento progressivo da frequência e intensidade dos espasmos, levando o indivíduo a ter cada vez mais limitações em suas atividades diárias ${ }^{(1)}$. O blefaroespasmo essencial benigno (BEB) e o espasmo hemifacial (EHF) são as distonias faciais mais frequentes.

O blefaroespasmo é uma disfunção muscular de etiologia ainda desconhecida. Faz parte do grupo de discinesias craniocervicais, caracterizado por movimentos involuntários da face, língua, palato, faringe, cordas vocais e pescoço. Pode aparecer de forma isolada, com acometimento dos músculos periorbitários (m. orbicular, m. prócero e m. corrugador), sendo chamado de blefaroespasmo essencial benigno (BEB), ou fazer parte de outras síndromes como Meige ou Brüeghel. O BEB é bilateral em $88 \%$ dos $\operatorname{casos}^{(1)}$. Muitas vezes as contrações se iniciam em um grupo muscular, espalhando-se para grupos adjacentes com o passar do tempo ${ }^{(2)}$.

O EHF caracteriza-se por contrações involuntárias unilaterais dos músculos inervados pelo VII par craniano. Possui etiologia distinta das síndromes mencionadas anteriormente, visto estar relacionado à estimulação do VII nervo próximo à sua origem. Diferentemente do BEB, pode persistir durante o sono e frequentemente cursa com enfraquecimento da musculatura facial subjacente ${ }^{(3)}$. É melhor classificado no grupo das mioclonias $^{(4)}$.

Os diagnósticos de BEB e de EHF exigem exames oftalmológico e neurológico completos ${ }^{(3)}$. Deve ser feito diagnóstico diferencial com: blefaroespasmo reflexo (secundário a irritações corneanas provocadas por triquíase, entrópio, blefarite e olho seco), fotofobia secundária a uveíte anterior ou catarata subcapsular posterior, secundário a drogas, associado a doenças neurológicas, miocmia do m. orbicular, apraxia palpebral, histeria $^{(2,3)}$.

Atualmente, o tratamento mais seguro e eficaz consiste em aplicações perioculares de toxina botulínica tipo A (BTX A), uma proteína de alto peso molecular que causa paralisia muscular pelo bloqueio pré-sináptico na placa motora ${ }^{(5,6)}$.

Apesar do considerável benefício gerado pelas aplicações de BTX A, muitos pacientes queixam-se da dor durante as injeções. Alguns métodos têm sido tentados para minimizar a dor. $\mathrm{O}$ uso de pomada anestésica como EMLA ${ }^{\circledR}$ tem demonstrado eficácia em alguns estudos ${ }^{(7,8)}$. Desde a antiguidade, gelo e neve são utilizados em medicina para combater febre, dor relacionada a gota e queimaduras. Atualmente, a crioterapia tem se mostrado indispensável em diversos tratamentos nas áreas de reumatologia, ortopedia, medicina esportiva e neurologia. ${ }^{(9)}$ A crioanestesia tem ganhado cada vez mais importância na dermatologia, tanto por promover anestesia da pele para diversos tratamentos a laser, como proteção térmica, permitindo maiores níveis de energia aplicados ${ }^{(9,10)}$. O resfriamento da pele tem se mostrado eficaz em produzir anestesia, sendo utilizado como jatos de ar frio, gelo, dentre outros ${ }^{(9,11)}$.

O objetivo deste estudo é avaliar a eficácia do resfriamento da pele com gelo no alívio da dor desencadeada pela injeção de toxina botulínica tipo A na região periocular em pacientes portadores de distonia facial.

\section{Métodos}

Estudo prospectivo, realizado no setor de Plástica Ocular da disciplina de oftalmologia da Faculdade de Medicina do ABC (FMABC). Foram incluídos 13 pacientes portadores de distonia facial tratados com aplicação de toxina botulínica tipo A (Prosigne $^{\circledR}$ - fabricado por Lanzhou Institute of Biological Products - China - importado pela Cristália) na região periocular. Tanto os pacientes portadores de BEB como de EHF foram submetidos à aplicação bilateral de BTX-A na região glabelar (Figura 1). No primeiro grupo o objetivo foi controlar o espasmo e no segundo controlar o espasmo ipsilateral e reduzir as rítides contralaterais.

Os critérios de exclusão foram: uso crônico de analgésicos ou ansiolíticos, neuropatias centrais ou periféricas, antecedente pessoal de alergia aos componentes da BTX A ou aos da pomada Epitezan ${ }^{\circledR}$.

Cada participante foi devidamente orientado sobre o estudo e assinou o termo de consentimento livre e esclarecido de forma voluntária. O estudo foi revisado e aprovado pelo Comitê de Ética em Pesquisa da Faculdade de Medicina do ABC.

A avaliação da eficácia do resfriamento da pele com gelo como anestésico foi realizada na região glabelar. Antes das injeções, o lado direito recebeu aplicação de gelo por 5 minutos e no outro lado foi aplicada pomada Epitezan ${ }^{\circledR}$ (pomada oftálmica acetato de retinol 10.000 UI, aminoácidos $2,5 \%$, metionina $0,5 \%$, cloranfenicol 0,5\%, da Allergan Produtos Farmacêuticos Ltda.), funcionando como placebo.

Observou-se eritema local após aplicação do gelo em todos os pacientes. Logo após a retirada da bolsa de gelo foi feita antissepsia da pele e retirada da pomada Epitezan ${ }^{\circledR}$ do lado oposto com álcool a 70\%, e aplicada BTX-A pela técnica de Brow, iniciando-se pelo lado que recebeu gelo.

Após a aplicação em cada lado o paciente era solicitado a dar uma nota para a dor sentida, numa escala de 0 (zero) a 10 (dez), sendo 0 ausência completa de dor e 10 a dor mais intensa.

Para análise estatística dos resultados foi utilizado um teste de hipóteses onde considerou-se a hipótese nula $\left(\mathrm{H}_{0}\right)$ como aquela que apresenta média das diferenças igual a zero $(0)$ e desvio padrão um (1), em uma curva normal (Figura 2). Como hipótese alternativa $\left(\mathrm{H}_{\mathrm{A}}\right)$ foram consideradas a média e o desvio padrão da amostra coletada. A hipótese nula $\mathrm{H}_{0}$ enuncia a

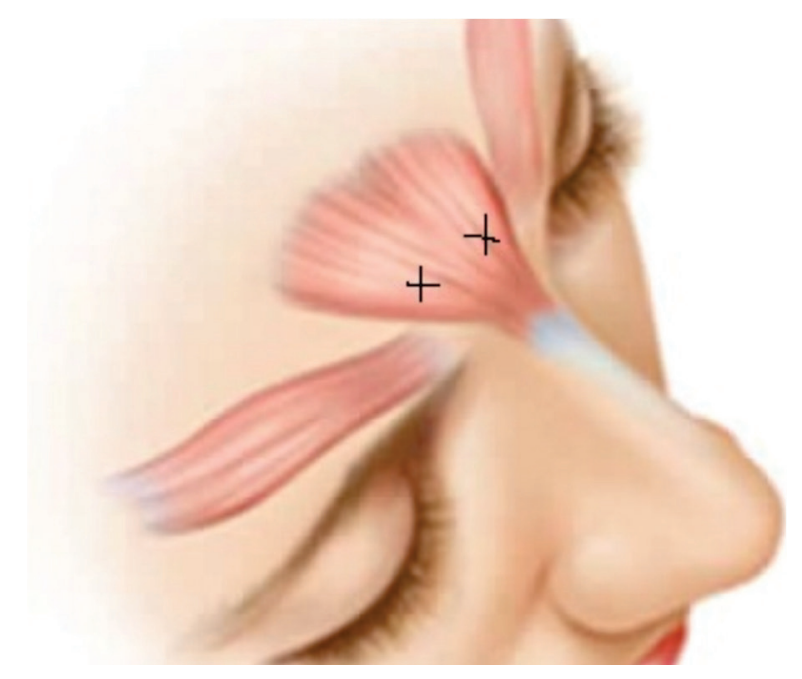

Figura 1: Locais de injeção da toxina botulínica na região glabelar 


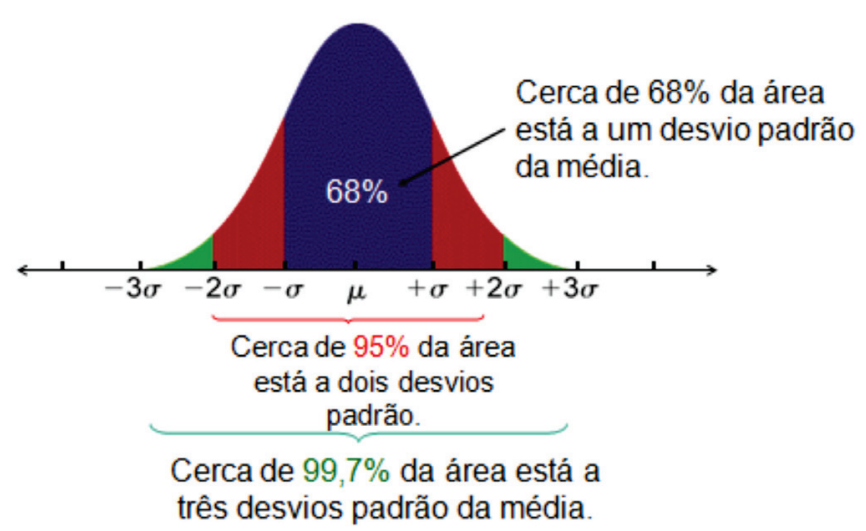

Figura 2: Representação gráfica da curva normal e a variação do desvio padrão em relação à média

afirmação contra a qual procuramos evidência, que seria não haver diferença entre usar ou não o gelo para analgesia. A estatística de teste mede o quanto os dados amostrais divergem da hipótese nula (resultados que gerassem média zero, ou seja, média zero para as diferenças de notas dadas pelos pacientes para a dor). Se a estatística de teste estiver na direção sugerida pela hipótese alternativa $\mathrm{H}_{\mathrm{A}}$, temos dados que seriam improváveis se $\mathrm{H}_{0}$ fosse verdadeira. A probabilidade calculada supondo-se que $\mathrm{H}_{0}$ é verdadeira, de que a estatística de teste assumisse um valor tão ou mais extremo do que o valor realmente observado, é chamada de $p$-valor. Quanto menor o $p$-valor, mais forte a evidência contrária à $\mathrm{H}_{0}$ fornecida pelos $\operatorname{dados}^{(12)}$.

\section{Resultados}

Dos 13 pacientes estudados, 4 eram portadores de BEB e 9 de EHF. Ambos os grupos foram submetidos a injeção bilateral de BTX-A na região periocular e glabelar

A idade dos pacientes que participaram deste estudo variou entre 59 e 89 anos, sendo a média das idades $71,15 \pm 10,6$. (Tabela 1).

As notas dadas pelos pacientes para graduar a dor sentida após as aplicações estão demonstradas na tabela 1. A média das notas dadas pelos pacientes à dor da injeção do lado onde foi aplicado placebo foi 3,92 $\pm 3,28$. No local onde foi aplicado gelo a média de notas de dor foi de 2,92 $\pm 2,18$.

A média de $\mathrm{H}_{\mathrm{A}}$ (média da diferença entre as notas do lado com e sem resfriamento) foi de 1,0 (Tabela 1). O desvio padrão da média das diferenças entre notas $(1,0)$ foi de 1,68 . Sabendose que o desvio padrão da população (DP ou $\boldsymbol{\sigma}$ ) é igual ao desvio padrão da amostra dividido pela raiz de n (neste estudo, treze amostras), então:

$$
\mathbf{D P}=1,68 / \sqrt{ } 13 ; \mathrm{DP}=1 / 3,61=0,465 .
$$

Chamando-se de $\mathbf{Z}$ o coeficiente de probabilidade em uma curva normal, $\bar{x}$ a média de $\mathrm{H}_{\mathrm{A}}$ (média das notas dos pacientes, a hipótese alternativa) e $\boldsymbol{\mu}$ (a média de $\mathrm{H}_{0}$ ou hipótese nula, onde a média das diferenças entre as notas seria zero caso as notas entre gelo e placebo fossem iguais), temos a equação da curva normal que define $\mathbf{Z}=\bar{x}-\boldsymbol{\mu} / \boldsymbol{\sigma}$; onde $\mathrm{Z}$ será o quociente entre a diferença entre a média de $\mathrm{H}_{\mathrm{A}}$ e a média de $\mathrm{H}_{0}$ e o desvio padrão. Desta forma temos:

$$
\mathbf{Z}=(1,0-0) / 0,465=1 / 0,47=2,13
$$

Tabela 1 Idades dos pacientes, diagnóstico e nota da dor referida

\begin{tabular}{cccccc}
\hline Paciente & Idade & Diagnóstico & $\begin{array}{c}\text { Nota lado } \\
\text { placebo }\end{array}$ & $\begin{array}{c}\text { Nota lado } \\
\text { gelo }\end{array}$ & $\begin{array}{c}\text { Diferença } \\
\text { entre notas }\end{array}$ \\
\hline 1 & 64 & EHF & 10 & 7 & 3 \\
2 & 61 & BEB & 7 & 5 & 2 \\
3 & 83 & EHF & 0 & 0 & 0 \\
4 & 60 & EHF & 1 & 2 & -1 \\
5 & 89 & EHF & 7 & 5 & 2 \\
6 & 76 & EHF & 6 & 3 & 3 \\
7 & 73 & EHF & 6 & 5 & 1 \\
8 & 75 & BEB & 2 & 4 & -2 \\
9 & 82 & EHF & 0 & 0 & 0 \\
10 & 60 & BEB & 2 & 1 & 1 \\
11 & 59 & BEB & 5 & 3 & 2 \\
12 & 63 & EHF & 0 & 1 & -1 \\
13 & 80 & EHF & 5 & 2 & 3 \\
\hline Média & 71,15 & & 3,92 & 2,92 & 1,0 \\
\hline
\end{tabular}

Na prática, o valor de $\mathbf{Z}$ ser 2,13 significa que encontramos a média das diferenças de percepção de dor 2,13 vezes maior que o desvio padrão na curva normal, que é a unidade. Muito raramente encontraríamos esse valor se de fato não existisse diferença nos procedimentos ${ }^{(13)}$.

Consultando a tabela normal temos, para o valor de $\mathbf{Z}=$ 2,13 , que a probabilidade da média das diferenças de percepção de dor é maior que 0,9834 . Sendo o $p$-valor a probabilidade de $\mathrm{H}_{0}$ ser verdadeira, fazemos seu cálculo por $1-0,9998=0,0166$. Assim sendo, o $p$-valor ser 0,0166 nos diz que é muito improvável que a anestesia com gelo seja ineficaz, ou seja, a probabilidade da analgesia com gelo ser ineficaz seria de $1,66 \%$.

\section{Discussão}

Segurança e conforto no tratamento são fundamentais para a adesão terapêutica, assim como a eficácia, custo e disponibilidade dos medicamentos envolvidos. Dor e estresse desencadeados por injeções repetidas podem gerar um desconforto tamanho que ocasione abandono do tratamento. ${ }^{(11)}$ Diversos autores têm sugerido aplicação de EMLA ${ }^{\circledR}$ para minimizar a dor da injeção de toxina botulínica ${ }^{(11,14)}$, de punções venosas, ${ }^{(15-17)}$ lombares ${ }^{(18)}$, acessos arteriais ${ }^{(19)}$ e procedimentos dermatológicos superficiais ${ }^{(11,20,21)}$. Porém, a necessidade de contato da pomada com a pele por 60 minutos torna o método pouco prático. Alguns autores utilizam lidocaína e epinefrina para diluir a BTX, mas este método atua apenas na redução da dor durante a injeção do medicamento, não atuando na punctura da pele com a agulha ${ }^{(11,21)}$

O poder anestésico, analgésico e protetor térmico do resfriamento tem sido largamente empregado em procedimentos médicos, mostrando-se um método efetivo na prevenção da dor durante injeção(22-26). Os receptores de dor são terminações nervosas livres, bastante prevalentes em camadas superficiais da pele. Desta forma, quando uma agulha, ainda que fina, é inserida na pele, há dor aguda. O frio previne a percepção da dor através de seu efeito nos nociceptores, diminuindo o tempo de condução e a atividade sináptica em nervos periféricos. ${ }^{(11)}$

No estudo de Linder JS et al. ${ }^{(10)} \mathrm{o}$ efeito anestésico do gelo foi testado antes da injeção de BTX A, assim como no estudo desenvolvido por Elibol $\mathrm{O}$ et al. ${ }^{(11)}$ Ambos obtiveram resultados favoráveis à aplicação do gelo, este com $p<0,001^{(11)}$. 
No presente estudo houve grande desvio padrão nas notas finais, provavelmente devido à diferença de sensibilidade à dor para cada paciente. Assim, um paciente pouco sensível à dor como o paciente \#13 deu nota 5 para o lado placebo e 2 para o lado gelo, resultando em uma diferença de 3. Já o paciente \#1, por exemplo, mostrou-se mais sensível à dor atribuindo nota 10 para o lado placebo e 7 para o do gelo. Nota-se neste caso notas muito mais altas, gerando alto desvio padrão das notas, mas mantendo a diferença de 3 entre os dois lados. Esta observação torna o estudo coerente, já que o resultado esperado foi observado nos dois casos (e na amostra total, $\operatorname{com} p<0,0166$ ), visto ter havido redução da dor proporcionalmente semelhante nos dois casos após aplicação de gelo local.

\section{Conclusão}

No presente estudo o resfriamento da pele com gelo foi eficaz no alívio da dor desencadeada pela aplicação de toxina botulínica tipo A na região periocular em pacientes portadores de distonia facial.

\section{REFERÊNCIAS}

1. Osako M, Keltner JL. Botulinum A toxin (Oculinum) in ophthalmology. Surv Ophthalmol. 1991;36(1):28-46.

2. Dutton JJ, Buckley EG. Long-term results and complications of botulinum A toxin in the treatment of blepharospasm. Ophthalmology. 1988;95(11):1529-34.

3. Nesi FA, Lisman RD, Levine MR. Smith's ophthalmic plastic and reconstructive surgery. 2nd ed. St. Louis: Mosby; 1998.

4. Villas Boas MLMM, Álvares MG, Moura EM. Blefaroespasmo. In: Soares EJC, Moura EM, Gonçalves JOR. Cirurgia plástica ocular. São Paulo: Roca; 1997. p. 203-19.

5. Carruthers A, Carruthers J. Toxina botulínica. 2a ed. Rio de Janeiro: Elsevier; 2009.

6. Perman KI, Baylis HI, Rosenbaum AL, Kirschen DG. The use of botulinum toxin in the medical management of benign essential blepharospasm. Ophthalmology. 1986;93(1):1-3.

7. Lander J, Hodgins M, Nazarali S, McTavish J, Ouellette J, Friesen E. Determinants of success and failure of EMLA. Pain. 1996;64(1):89-97.

8. Söylev MF, Koçak N, Kuvaki B, Ozkan SB, Kir E. Anesthesia with EMLA cream for botulinum A toxin injection into eyelids. Ophthalmologica. 2002;216(5):355-8.

9. Raulin C, Greve B, Hammes S. Cold air in laser therapy: first experiences with a new cooling system. Lasers Surg Med. 2000;27(5):404-10.

10. Linder JS, Edmonson BC, Laquis SJ, Drewry RD Jr, Fleming JC. Skin cooling before periocular botulinum toxin A injection. Ophthal Plast Reconstr Surg. 2002;18(6):441-2.

11. Elibol O, Ozkan B, Hekimhan PK, Caglar Y. Efficacy of skin cooling and EMLA cream application for pain relief of periocular botulinum toxin injection. Ophthal Plast Reconstr Surg. 2007;23(2):130-3.
12. McCord CD Jr, Coles WH, Shore JW, Spector R, Putnam JR Treatment of essencial blepharospasm. I. Comparison of facial nerve avulsion and eyebrow-eyelid muscle stripping procedure. Arch Ophthalmol. 1984;102(2):266-8.

13. Moore DS. A estatística básica e sua prática. 3a ed. Rio de Janeiro: Livros Técnicos e Científicos; 2005.

14. Dutton JJ. Botulinum-A toxin in the treatment of craniocervical muscle spasms: short- and long-term, local and systemic effects. Surv Ophthalmol. 1996;41(1):51-65.

15. Hallén B, Carlsson P, Uppfeldt A. Clinical study of a lignocaineprilocaine cream to relieve the pain of venepuncture. Br J Anaesth. 1985;57(3):326-8.

16. Maunuksela EL, Korpela R. Double-blind evaluation of a lignocaineprilocaine cream (EMLA) in children. Effect on the pain associated with venous cannulation. Br J Anaesth. 1986;58(11):1242-5.

17. Hopkins CS, Buckley CJ, Bush GH. Pain-free injection in infants. Use of a lignocaine-prilocaine cream to prevent pain at intravenous induction of general anaesthesia in 1-5-year-old children. Anaesthesia. 1988;43(3):198-201.

18. Kapelushnik J, Koren G, Solh H, Greenberg M, DeVeber L. Evaluating the efficacy of EMLA in alleviating pain associated with lumbar puncture; comparison of open and double-blinded protocols in children. Pain. 1990;42(1):31-4.

19. Smith M, Gray BM, Ingram S, Jewkes DA. Double-blind comparison of topical lignocaine-prilocaine cream (EMLA) and lignocaine infiltration for arterial cannulation in adults. Br J Anaesth. 1990;65(2):240-2.

20. Sunderraj P, Kirby J, Joyce PW, Watson A. A double-masked evaluation of lignocaine-prilocaine cream (EMLA) used to alleviate the pain of retrobulbar injection. Br J Ophthalmol. 1991;75(3):1302. Comment in Br J Ophthalmol. 1991;75(3):129.

21. Gotsis SS, Volonaki OM, Theodossiadis GP. Percutaneous anesthesia with a lignocaine-prilocaine cream (EMLA) for eyelid skin surgery. Br J Ophthalmol. 1994;78(3):209-10.

22. Gassner HG, Sherris DA. Addition of an anesthetic agent to enhance the predictability of the effects of botulinum toxin type A injections: a randomized controlled study. Mayo Clin Proc. 2000;75(7):701-4.

23. Fisher GH, Kim KH, Bernstein LJ, Geronemus RG. Concurrent use of a handheld forced cold air device minimizes patient discomfort during fractional photothermolysis. Dermatol Surg. 2005;31( Pt 2):1242-3; discussion 1244.

24. Altshuler GB, Zenzie HH, Erofeev AV, Smirnov MZ, Anderson RR, Dierickx C. Contact cooling of the skin. Phys Med Biol. 1999;44(4):1003-23.

25. Ernst E, Fialka V. Ice freezes pain? A review of the clinical effectiveness of analgesic cold therapy. J Pain Symptom Manage. 1994;9(1):56-9.

26. Kuzu N, Ucar H. The effect of cold on the occurrence of bruising, haematoma and pain at the injection site in subcutaneous low molecular weight heparin. Int J Nurs Stud. 2001;38(1):51-9.

\section{Autor correspondente:}

Nilson Lopes da Fonseca Junior

Avenida Ibirapuera, no 2907- conj.1601- Moema

CEP 04029-200 - São Paulo (SP), Brasil

E-mail: lopesdafonseca@uol.com.br 